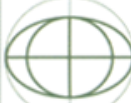

KN Kartographische Nachrichten Journal of Cartography and Geographic Information 65. Jahrgang, Oktober 2015, Heft 5 Herausgeber: Deutsche Gesellschaft für Kartographie e.V. (DGfK) - Gesellschaft für Kartographie und Geomatik vertreten durch den Präsidenten Prof. Dr. Manfred Weisensee

Organ der Deutschen Gesellschaft für Kartographie e.V. (DGfK). der Schweizerischen Gesellschaft für Kartografie (SGK) und der Osterreichischen Kartographischen Kommission (OKK) in der Osterreichischen Gesellschaft für Geographie (OGG).

Schriftleitung

Hauptschriftleiter

Prof. Dr. rer. nat. habil. Mark Vetter (zustāndig fûr Aufsătze, Berichte). Hochschule Karlsruhe - Technik und Wirtschaft, Moltkestr. 30, 76133 Karlsruhe; redaktion@kartographischenachrichten.de, fon: 0049(0) 7219252599

Weitere Schriftleiter

Prof. Dr. phil. Frank Dickmann (Zweiter Schriftleiter, zustăndig fût Nachrichten aus Hochschulen und Institutionen, Rezensionen). Ruhr-Universităt Bochum, Geographisches Institut, Postfach, 44780 Bochum; frank.dickmann@rub.de Fon: 0049(0) 234 32-23379; Fax: 0049(0) $23432-14964$ Dipl.-Ing. Andreas Gollenstede (zustãndig für Geoinformation aktuell) Haareneschstr. 91, 26121 Oldenburg: kn@gollenstede.com Fon: 0049(0) 441 7779-545; Fax: 0049(0) 441 7779.750 Dr. Thomas Chudy (zuständig für DGfK-, SKG- und OGG. Nachrichten). Martin-Luther-Universităt Halle-Wittenberg, Institut für Agrar- und Ernăhrungswissenschaften, Karl-Freiherr-vonFritsch-Straße 4, 06120 Halle/Saale, fon: 0049(0) 3455522448 ; Fax: 0049(0) 3455527109

Prof. Dr. Reinhard Zōlitz (zustăndig für Nachrichten aus Hochschulen und Institutionen), Institut für Geographie und Geologie. Kartographie und GIS, Friedrich-Ludwig-Jahn-Str. 16, D-17489 Greifswald, Fon 03834864523 , Fax: 03834864501 .

E-Mail: zoelitz@uni-greifswald.de

Mitarbeit für die Schweiz: Prof. Dr. Lorenz Hurni. Institut für Kartografie, ETH Hōnggerberg, 8093 Zürich; hurni@karto.baug. ethz.ch. Fon: 004116333033 ; Fax: 00411631153

Mitarbeit für Osterreich: Univ.-Prof. Dr. Wolfgang Kainz. Institut für Geographie und Regionalforschung, Universităt Wien,

Universitätsstraße 7/5, 1010 Wien; wolfgang-kainz @univie. ac at

Fon: 0043 14277-8640; Fax: 0043 14277-9531

Ehrenschriftleiter

Prof i. R. Dr Jürgen Dodt, Witten

Dr. rec nat h. . Rolf Harbeck. Bonn

Editorial Board

Prof. Dr. H. Asche, Potsdam; Prof. Dr. Manfred Buchroithner, Dresden: Prof i. R. Dr. Jürgen Dodt, Bonn; Prof. Dr. D. Dransch, Potsdam; Prof. Dr. M. Ehlers, Osnabrück, Berlin; Prof. Dr. S. I. Fabrikant, Zürich, Schweiz; Ass. Prot. Dr. S. Fuhrmann, Fairfax, USA: Prot. Dr. G. Gartner: Wien, Osterreich; Prof. Dr. D. Grünreich, Ronnenberg; Dr. Rolf Harbeck, Bonn; Prof. Dr. A. Hüttermann, Ludwigsburg; Prof. Dr. L. Hurni, Zürich; Prof. Dr.W. Kainz, Wien; Prof. Dr. P. Kammerer, München

Prot. Di. W. Kainz, Wien, Prof. Dr. P. Kammere, München,

Prof. Dr. J. Schiewe, Hamburg; PD Dr.-Ing. J. Schoppmeyer, Bonn;

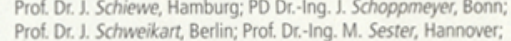
Prof. Dr. J. Schweikart, Berlin; Prof. Dr.-Ing. M. Sester, Hannover;
Prof. Dr. J. Siemer, Regina, Kanada; Prof. Dr. R. Zólitz, Greifswald. Manuskripte richten Sie bitte an den zuständigen Schriftleiter. Hinweise zur Gestaltung des Manuskripts finden Sie unter www.kartographische-nachrichten.de > Autorenhinweise. Reviewverfahren

Wissenschaftliche Beiträge werden im Rahmen eines Peer-ReviewVerfahrens begutachtet. Trotz sorgfáltiger Redigierung übernehmen Herausgeber Redaktion und Verlag keine Haftung für die inhalttiche Richtigkeit der Veröffentlichungen.

Die KN Kartographische Nachrichten ist in der internationalen Zitationsdatenbank Scopus gelistet.

\section{Die DGfK ist Mitglied der}

Internationalen Kartographischen

Vereinigung IKV/CA.

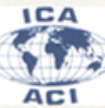

Verlag: Kirschbaum Verlag GmbH, SiegfriedstraBe 28,

D.53179 Bonn / Postfach 210209, D.53157 Bonn.

Fon: (0228) 95453-0. Fax: (0228) 95453-27:

Internet: http://www.kirschbaum.de, E-Mail: info@kirschbaum.de

Anzeigenleiter: Volker Rutkowski; zurzeit ist Anzeigenpreisliste

Nr. 50 vom 1.10 .2014 gültig

Bezugspreise und ISSN: Inland 67,70 Euro jāhrlich, inkl. MwSt. und Versandkosten; Einzelheft 12,- Euro inkl. MwSt.

Ausland 69,80 Euro jährlich, inkl. MwSt. und Versandkoster: Einzelheft 12,- Euro inkl. MwSt.

Die Zeitschrift erscheint alle zwei Monate. ISSN 0022-9164.

Satz: Mohr Mediengestaltung, Siebengebirgsweg 40, D-53773 Hennef Druck: SDV Saarländische Druckerei und Verlag GmbH, Werner-yon-Siemens-Straße 31, D-66793 Saanwellingen

\title{
Bildung über Karten
}

Diese Ausgabe der Kartographischen Nachrichten erscheint anlässlich des DKG, des Deutschen Kongresses für Geographie, der in Berlin vom 1.-6. Oktober 2015 stattfindet. Nach alter Zählung ist dies gleichzeitig der 59. Deutsche Geographentag, eine Tradition, die seit dem Jahr 1865 besteht. Die im vorliegenden Heft dokumentierte Liaison zwischen Kartographie und Geographie liegt in diesem speziellen Fall im Wesentlichen daran, dass der Schriftleiter als Geograph dort zusammen mit seinem Kollegen Dr. Friedrich Barnikel, Fachkoordinator Geographie für Städtische Gymnasien in München, als Leiter einer Session vertreten sein wird. Das Thema dieser Session ist: „Von der analogen zur digitalen Karte - Der fundamentale Wandel der Kartographie”. Wir sind gemeinsam der festen Überzeugung, dass die Verbindung zwischen Kartographie und Geographie nicht nur immer schon geschwisterlich war, sondern dies in Zukunft auch bleiben wird. Die beiden Disziplinen stehen sinnvollerweise im fruchtbaren Austausch, sie benötigen einander! Dies lässt sich schon alleine daran dokumentieren, dass viele Geographinnen und Geographen Artikel für die $\mathrm{KN}$ verfassen, der geneigte Leser mag nur einen Blick in die Hefte der vergangenen Jahre werfen.

Geographie findet professionell, außerhalb forschender Hochschulen und anwendungsorientierter

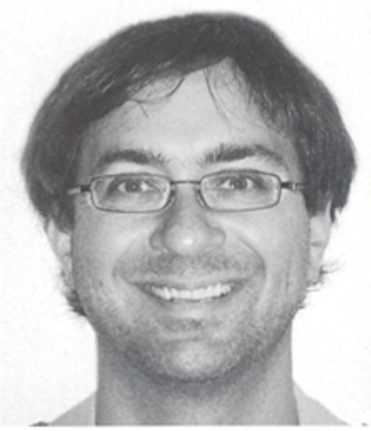

Friedrich Barnikel

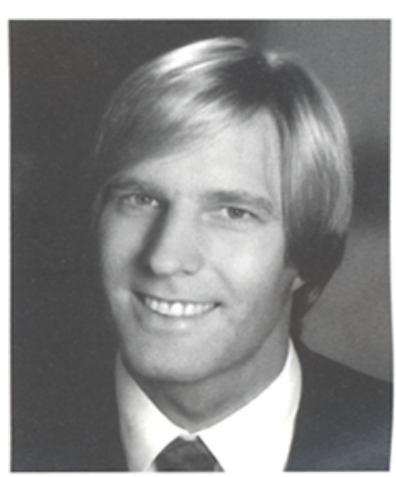

Mark Vetter

Firmen, vor allem in der Schule statt. Die Schule, bzw. der Geographie-/Erdkundeunterricht, setzt verschiedene Geomedien analog und digital ein und lehrt jungen Menschen mit Globen, Atlanten oder mit einzelnen thematischen Karten zu arbeiten. Didaktikerinnen und Didaktiker der Geographie und gleichermaßen die Lehrerinnen und Lehrer der Geographie und verwandter Fächer in der ganzen Welt, können ihre Arbeit unmöglich ohne Karten leisten, ganz gleich ob diese analoger oder digitaler Natur sind. Karten sind im besten Sinne des Wortes Bildungsinstrumente. Und die (Aus-)Bildung zum kritischen Menschen findet auch ein stückweit über Karten statt.

Die Beiträge im vorliegenden Heft zeigen dies exemplarisch. Das Autorenteam um Dennis Edler stellt in dem Beitrag vor, wie die „lebendige Karte” als audiovisuelles Instrument im Unterricht eingesetzt werden kann. Florian Hruby skizziert die Transformation von der analogen zur digitalen Karte unter Berücksichtigung des Bildungsaspektes. Digitale Atlanten sind nicht mehr allein Produkt, sondern vielmehr vielfach bereits partizipativer Prozess. Frank Dickmann, Dennis Edler, Anne-Kathrin Bestgen und Lars Kuchinke untersuchen kartenexperimentell die Blickbewegungen von Kartennutzern und ihre Beeinflussung durch kartographische Elemente und visualisieren die Ergebnisse in „Heatmaps" Mateusz Czaja designt anamorphe Stadtpläne. Als Beispiel stellt er Wrocław/Breslau vor. Lisa Paul und Getrud Schaab zeigen, wie man mit Karten spielerisch ein nachhaltiges Verhalten vermitteln kann. In ihrem Beitrag werden sozusagen Geomedien als Mittel der Umweltbildung verwendet. Zu guter Letzt stellen Ronny Schomacker, Eduard Gert und Jürgen Schweikart eine Anwendung vor, die es Nutzern erlaubt, Kunst im öffentlichen Raum nach Attributen geordnet zu lokalisieren.

Wir wünschen Ihnen viel Freude beim Lesen der Beiträge und freuen uns auf eine Begegnung auf dem DKG in Berlin!

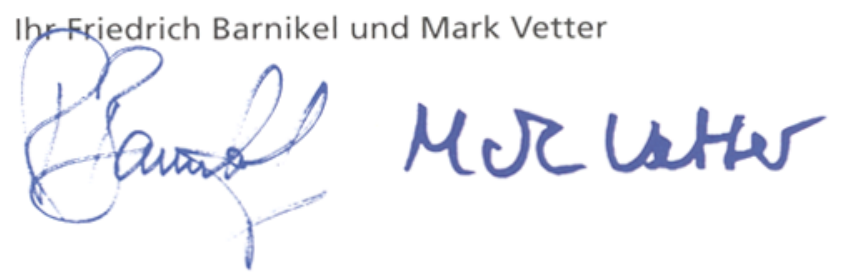

